\title{
Urinary Apolipoprotein C3 Is a Potential Biomarker for Alzheimer's Disease
}

\author{
Yumi Watanabe $^{a}$ Yoshitoshi Hirao $^{\mathrm{b}}$ Kensaku Kasuga $^{\mathrm{c}}$ \\ Takayoshi Tokutake $^{d}$ Kaori Kitamura $^{a}$ Shumpei Niida ${ }^{e}$ Takeshi Ikeuchi $^{c}$ \\ Kazutoshi Nakamura ${ }^{a}$ Tadashi Yamamoto ${ }^{b, f}$ \\ ${ }^{a}$ Division of Preventive Medicine, Niigata University Graduate School of Medical and Dental \\ Sciences, Niigata, Japan; 'biofluid Biomarker Center, Niigata University Graduate School

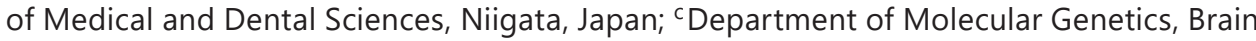 \\ Research Institute, Niigata University, Niigata, Japan; dDepartment of Neurology, Brain \\ Research Institute, Niigata University, Niigata, Japan; ${ }^{e}$ Research Institute, National Center \\ for Geriatrics and Gerontology, Obu, Japan; ${ }^{f}$ Department of Clinical Laboratory, Shinrakuen \\ Hospital, Niigata, Japan
}

\section{Keywords}

Biomarker $\cdot$ Urine $\cdot$ Alzheimer's disease

\begin{abstract}
Introduction: Biomarkers of Alzheimer's disease (AD) that can easily be measured in routine health checkups are desirable. Urine is a source of biomarkers that can be collected easily and noninvasively. We previously reported on the comprehensive profile of the urinary proteome of $A D$ patients and identified proteins estimated to be significantly increased or decreased in $A D$ patients by a label-free quantification method. The present study aimed to validate urinary levels of proteins that significantly differed between AD and control samples from our proteomics study (i.e., apolipoprotein $\mathrm{C} 3$ [ApoC3], insulin-like growth factor-binding protein 3 [lgfbp3], and apolipoprotein D [ApoD]). Methods: Enzyme-linked immunosorbent assays (ELISAs) were performed using urine samples from the same patient and control groups analyzed in the previous proteomics study (18 AD and 18 controls, set 1 ) and urine samples from an independent group of $A D$ patients and controls (13 $A D, 5$ mild cognitive impairment $[\mathrm{MCl}]$, and 32 controls) from the National Center for Geriatrics and Gerontology Biobank (set 2). Results: In set 1 , the crude urinary levels of $A p o D$, Igfbp3, and creatinine-adjusted $A p o D$ were significantly higher in the AD group relative to the control group $(p=0.003, p=0.002$, and $p=0.019$, respectively), consistent with our previous proteomics results. In set 2 , however, the crude urinary levels of Igfbp3 were significantly lower in the $A D+\mathrm{MCl}$ group than in the con-
\end{abstract}


trol group ( $p=0.028$ ), and the levels of ApoD and ApoC3 did not differ significantly compared to the control group. Combined analysis of all samples revealed creatinine-adjusted ApoC3 levels to be significantly higher in the AD+MCl group $(p=0.015)$ and the AD-only group $(p=$ 0.011 ) relative to the control group. Conclusion: $A p o C 3$ may be a potential biomarker for $A D$, as validated by ELISA. Further analysis of $A p o C 3$ as a urinary biomarker for $A D$ is warranted.

(C) 2020 The Author(s)

Published by S. Karger AG, Basel

\section{Introduction}

Alzheimer's disease (AD) accounts for $60-70 \%$ of dementia cases and its control is important to decrease the prevalence of dementia [1,2]. Since no cure for AD is currently available, it is important to detect it early and to intervene, for example, with lifestyle modifications [3]. At present, combinations of cerebrospinal fluid biomarkers such as amyloid- $\beta 42$, total tau, and phosphorylated tau, and neurological imaging modalities such as structural magnetic resonance imaging and positron emission tomography are used to diagnose AD [4]. However, these tests are invasive and expensive, and so are unlikely to be applicable to the general population. Thus, there is an urgent need for AD biomarkers that can be screened easily and noninvasively.

Urine can be collected in a relatively large amount and frequently in a noninvasive manner. It contains a variety of biomolecules, and its composition changes depending on biological and pathological conditions. This makes urine ideal for biomarker discovery and screening tests [5]. However, studies on urinary AD biomarkers are limited [6], with only a few reports on protein biomarkers [7, 8].

We previously conducted a comprehensive study of the urinary proteome of $18 \mathrm{AD}$ patients and 18 cognitively normal controls by label-free liquid chromatography-tandem mass spectrometry [7]. Gene enrichment and molecular network analyses revealed the urinary proteome of $\mathrm{AD}$ patients to be enriched with proteins related to lysosomes, the complement pathway, and gluconeogenesis, as well as proteins involved in the canonical pathways of lipoprotein metabolism, HSP90 signaling, MMP signaling, and redox regulation by thioredoxin [7]. In that study, several proteins were identified as being increased or decreased in $\mathrm{AD}$ patients relative to controls.

In the present study, the urine levels of three candidate proteins identified in our prior urinary proteome analysis as differing in levels between $\mathrm{AD}$ and control samples (i.e., apolipoprotein C3 [ApoC3], insulin-like growth factor-binding protein 3 [Igfbp3], and apolipoprotein D [ApoD]) were measured by enzyme-linked immunosorbent assay (ELISA) and compared between $\mathrm{AD}$ and control groups.

\section{Materials and Methods}

\section{Participants}

Participants were divided into two sets (sets 1 and 2). Set 1 included 18 AD patients and 18 cognitively normal elderly people (controls) selected in an age- and sex-matched manner [7]. Briefly, AD patients visiting Niigata University Hospital who were diagnosed with the disease based on the criteria of the National Institute of Neurological and Communicative Disorders and Stroke Alzheimer's Disease and Related Disorders Association and who were aged between 60 and 80 years were recruited. Controls were selected in an ageand sex-matched manner from a subcohort (Sekikawa cohort) of the Murakami cohort, a population-based cohort study in areas of the northern Nigata prefecture [9]. Cognitive 
Table 1. Characteristics of the set 2 participants

\begin{tabular}{|c|c|c|c|c|}
\hline & $\mathrm{AD}(n=13)$ & $\operatorname{MCI}(n=5)$ & Controls $(n=32)$ & $p$ value \\
\hline Age, years & $79.9 \pm 6.6$ & $81.8 \pm 3.9$ & $71.9 \pm 8.4$ & $0.002^{\mathrm{a}}$ \\
\hline Male, $n(\%)$ & $4(30.8 \%)$ & $1(20 \%)$ & $16(50 \%)$ & $0.245^{c}$ \\
\hline MMSE, points & $17.1 \pm 7.9$ & $24.6 \pm 1.8$ & not available & $0.003^{\mathrm{b}}$ \\
\hline \multicolumn{5}{|l|}{ Results of urine test strip } \\
\hline Urine protein level, $n$ & $-(12), \pm(1)$ & $-(3), \pm(2)$ & $-(19), \pm(13)$ & $0.009^{c}$ \\
\hline Urine blood level, $n$ & $-(10),+(1), 2+(2)$ & $-(5)$ & $-(31),+(1)$ & $0.085^{c}$ \\
\hline Urine glucose level, $n$ & $-(13)$ & $-(13), 4+(1)$ & $-(16), \pm(4), 2+(1), 3+(2), 4+(1)$ & $0.005^{c}$ \\
\hline Type 2 diabetes, $n(\%)$ & $1(7.7 \%)$ & $2(40 \%)$ & $25(78 \%)$ & $<0.0001^{c}$ \\
\hline Urinary creatinine, $\mathrm{mg} / \mathrm{dL}$ & $74.3 \pm 35.2$ & $74.2 \pm 34.4$ & $101.8 \pm 55.7$ & $0.181^{\mathrm{a}}$ \\
\hline
\end{tabular}

Data are presented as mean \pm standard deviation for continuous variables. AD, Alzheimer's disease; MCI, mild cognitive impairment; MMSE, Mini-Mental State Examination. ${ }^{a}$ Analysis of variance. ${ }^{b}$ Fisher's exact test. ${ }^{c}$ Unpaired $t$ test with Welch's correction.

performance was assessed by the Japanese version of the Mini-Mental State Examination (MMSE), and scores $>27$ were considered cognitively normal [10]. Detailed characteristics of set 1 have been described previously [7]. Briefly, the mean ages of the AD and control groups were $72.9 \pm 5.6$ and $72.8 \pm 5.2$ years, respectively, and there were 8 males and 10 females in each group. The mean MMSE scores were $21.6 \pm 4.5$ and $28.8 \pm 0.7$, respectively $(p<0.001, t$ test).

Urine samples for set 2 were obtained from the National Center for Geriatrics and Gerontology (NCGG) Biobank (14 AD, 5 mild cognitive impairment [MCI], and 36 controls without neuropsychiatric disorders). Diseases were classified based on the International Statistical Classification of Diseases and Related Health Problems 10th revision. MMSE scores were also obtained from the NCGG Biobank for AD and MCI participants. We excluded from the analysis $1 \mathrm{AD}$ patient and 4 controls who were positive for proteinuria ( + and ++ ) by test strips. The characteristics of set 2 participants are provided in Table 1.

\section{Urine Sample Collection and Laboratory Tests}

The procedure for collection and storage of set 1 urine samples has been described previously [7]. For set 2, spot urine was collected and centrifuged at 3,500 rpm for 5 min (Kubota 4000 , Kubota Corp., Japan), aliquoted, stored at $-80^{\circ} \mathrm{C}$, and transported in dried ice. Samples were thawed at $37^{\circ} \mathrm{C}$ for $10 \mathrm{~min}$ and checked using urine test strips (Pretest 5bII, Wako, Japan). Urinary creatinine levels were measured by latex immunological nephelometry using a SPOTCHEM D-01 analyzer (SD-3810, Arkray Global Business, Inc., Japan). Urine samples were aliquoted in small volumes and stored at $-20^{\circ} \mathrm{C}$ until use.

\section{Proteomics}

In the present study, we selected candidate proteins based on the analysis data of our previous proteomics study [7] for further confirmation by ELISA. Detailed methods for mass spectrometry, label-free quantification, and statistical analysis were reported previously [7]. Briefly, set 1 urine samples were analyzed by label-free liquid chromatography-tandem mass spectrometry. The normalized spectral index $\left(\mathrm{SI}_{\mathrm{N}}\right)$, a label-free quantification method [11], was used to compare protein abundance between different samples. While our previous proteomics analysis [7] focused only on proteins that showed a $>2$-fold significant increase or decrease in $\mathrm{AD}$ patients relative to controls, the present study analyzed all proteins from the previous dataset that were significantly increased or decreased in AD patients relative to controls. The $\mathrm{SI}_{\mathrm{N}}$ table used in the analysis is provided in online supplementary Table $\mathrm{S} 1$ (for all online suppl. material, see www.karger.com/doi/10.1159/000509561). 
Table 2. Significantly increased or decreased proteins $(p<0.01)$ among those detected in all samples

\begin{tabular}{lllllll}
\hline Gene name & $\begin{array}{l}\text { Fold change } \\
\text { (AD/control) }\end{array}$ & $p$ value & FDR & $\begin{array}{l}\text { Detected in } \\
\text { AD samples, } n\end{array}$ & $\begin{array}{l}\text { Detected in } \\
\text { control samples, } n\end{array}$ & $\begin{array}{l}\text { Rank } \\
\text { in AD }\end{array}$ \\
\hline APOD & 1.79 & 0.001 & 0.048 & 18 & 18 & 12 \\
Rank in \\
control
\end{tabular}

Top three proteins are shown. $p$ values were calculated using the unpaired $t$ test with Welch's correction. AD, Alzheimer's disease; FDR, false discovery rate.

\section{Enzyme-Linked Immunosorbent Assay}

Commercial ELISA kits were used to measure urinary levels of ApoC3 (ELH-ApoC3, RayBiotech, Inc., USA), ApoD (3714-1HP, Mabtech AB, Sweden), and Igfbp3 (KIT10430, Sino Biological Inc., Beijing, China). Samples were tested in duplicate. A microplate reader (iMARK ${ }^{\mathrm{TM}}$, Bio-Rad Laboratories Inc., USA) was used to measure absorbance at $450 \mathrm{~nm}$. The Microplate Manager ${ }^{\circledR} 6$ Software was used for data analysis. Each ELISA result was normalized by creatinine level.

\section{Statistical Analysis}

SAS (SAS 9.13, SAS Institute Inc., Cary, NC, USA) was used for demographic analysis of set 2. Categorical variables were expressed as numbers and percentages and compared with Fisher's exact tests or one-way factorial analysis of variance. Continuous variables were expressed as mean \pm standard deviation and compared with the $t$ test with Welch's correction. The Prism 8 software (GraphPad Software LLC, USA) was used for statistical analyses of proteomics and ELISA results and for generating graphs. The $t$ test with Welch's correction was used to compare $\mathrm{SI}_{\mathrm{N}}$ values between the AD and control groups. Since the ELISA data did not pass the D'Agostino-Pearson omnibus normality test for both $\mathrm{AD}$ (or $\mathrm{AD}+\mathrm{MCI}$ ) and control groups, these groups were compared using the Mann-Whitney U test. $p<0.05$ (two-tailed) was considered statistically significant.

\section{Results}

We selected Igfbp3 and ApoC3 as targets from our previous proteomics data [7] for confirmation by ELISA, since they were the most significantly increased and decreased proteins, respectively, in AD patients relative to controls according to $p$ values of Welch's $t$ test (online suppl. Table S1). However, given the low level of these proteins in urine, fluctuations in measurement by mass spectrometry may have been substantial. Accordingly, we additionally included ApoD in the analysis, as this protein was detected in all samples and was significantly increased in AD patients (Table 2; online suppl. Table S1).

The results of the ELISA experiment for set 1 are shown in Figure 1. The crude levels of ApoC3, Igfbp3, and ApoD were significantly higher in the AD group relative to the control group. The levels of creatinine-adjusted ApoD were significantly higher and the levels of creatinine-adjusted Igfbp3 tended to be higher in the AD group relative to the control group. No difference was observed in the levels of ApoC3 between the AD and control groups. The ELISA results for Igfbp3 and ApoD were consistent with the proteomics data, but the levels of ApoC3 in urine measured by ELISA showed an opposite tendency than what was observed in the proteomics data. 
Dementia and

Geriatric Cognitive

Disorders Extra

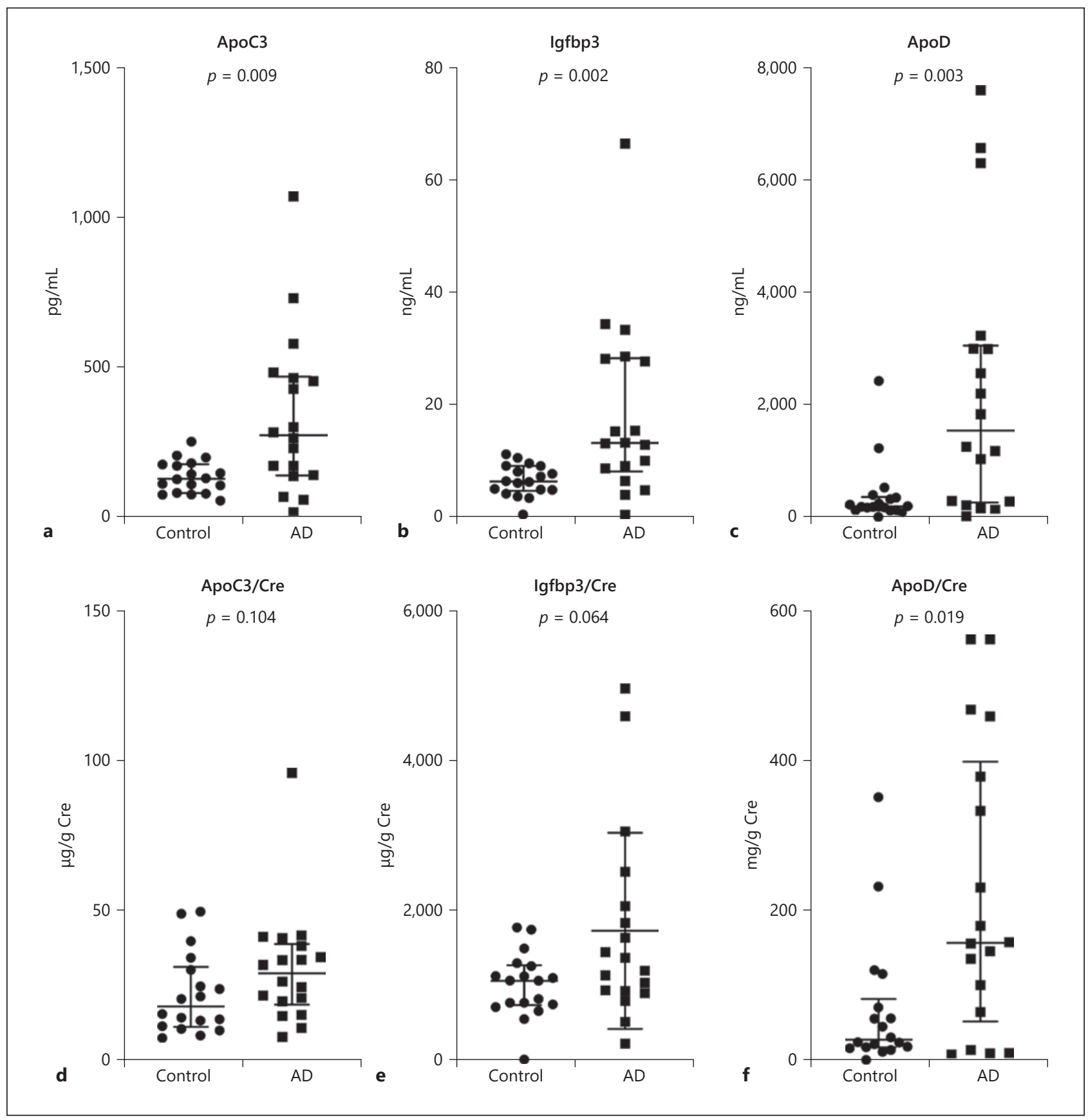

Fig. 1. Urinary ELISA of set 1. Urinary levels of ApoC3 (a, d), Igfbp3 (b, e), and ApoD (c, f) in set 1 (18 AD and 18 control urine samples from previous proteomics analysis) as measured by ELISA. Crude levels (a-c) and Cre-adjusted levels (d-f) of the three proteins. $p$ values were calculated using the Mann-Whitney U test. Data are presented as median and interquartile range. AD, Alzheimer's disease; ApoC3, apolipoprotein C3; ApoD, apolipoprotein D; Cre, creatinine; ELISA, enzyme-linked immunosorbent assay; Igfbp3, insulin-like growth factor-binding protein 3.

The results of the ELISA experiment for set 2 are shown in Figure 2. Given the limited size of the MCI population $(n=5), \mathrm{AD}$ and MCI were combined into one group for the analysis $(\mathrm{AD}+\mathrm{MCI}, n=19)$. The crude levels of Igfbp3 were significantly lower in the AD+MCI group relative to the control group, but no difference was found between the two groups after 
Dementia and

Geriatric Cognitive

Disorders Extra
Dement Geriatr Cogn Disord Extra 2020;10:94-104

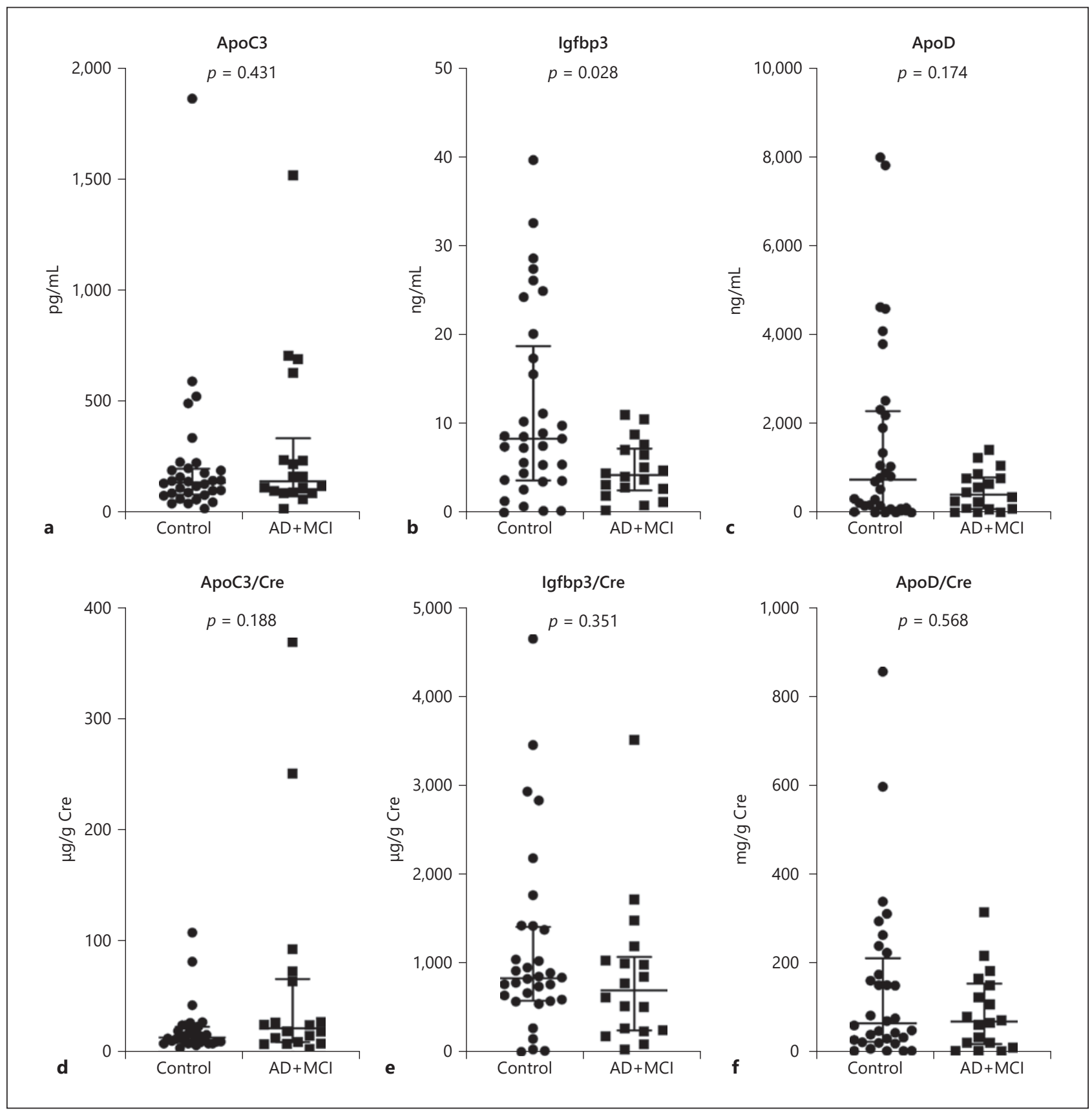

Fig. 2. Urinary ELISA of set 2. Urinary levels of ApoC3 (a, d), Igfbp3 (b, e), and ApoD (c, f) in set 2 (13 AD, 5 MCI, and 32 control urine samples) as measured by ELISA. Crude levels (a-c) and Cre-adjusted levels (d-f) of the three proteins. $p$ values were calculated using the Mann-Whitney U test. Data are presented as median and interquartile range. AD, Alzheimer's disease; ApoC3, apolipoprotein C3; ApoD, apolipoprotein D; Cre, creatinine; ELISA, enzyme-linked immunosorbent assay; Igfbp3, insulin-like growth factor-binding protein 3; MCI, mild cognitive impairment.

adjusting for creatinine. No difference was observed in both crude and creatinine-adjusted levels of ApoC3 and ApoD between the AD+MCI and control groups.

Since the same protein standards were used for all ELISA experiments, we also analyzed the results of sets 1 and 2 combined (Fig. 3; raw data and percent coefficient of variation of 
Dementia and

Geriatric Cognitive Disorders Extra
Dement Geriatr Cogn Disord Extra 2020;10:94-104

Watanabe et al.: Urinary ApoC3 as a Biomarker for Alzheimer's Disease

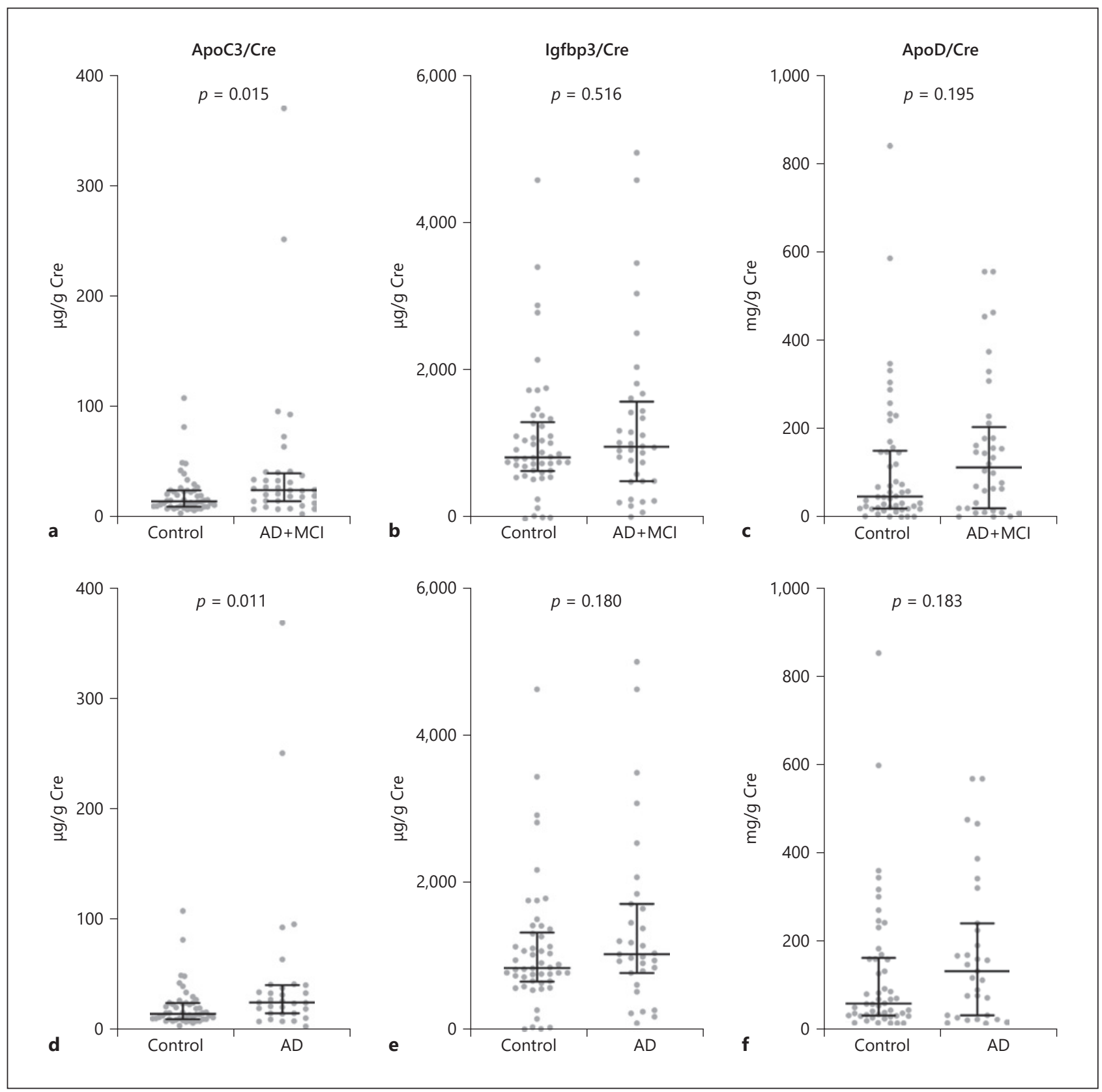

Fig. 3. Combined data from ELISAs of sets 1 and 2. Cre-adjusted levels of ApoC3 (a, d), Igfbp3 (b, e), and ApoD (c, f). a-c The AD+MCI group $(n=31+5)$ was compared with the control group $(n=50)$. d-f Results from 31 $\mathrm{AD}$ and 59 control samples. $p$ values were calculated using the Mann-Whitney U test. Data are presented as median and interquartile range. AD, Alzheimer's disease; ApoC3, apolipoprotein C3; ApoD, apolipoprotein D; Cre, creatinine; ELISAs, enzyme-linked immunosorbent assays; Igfbp3, insulin-like growth factor-binding protein 3; MCI, mild cognitive impairment.

each standard dilution are provided in online suppl. Table S2). The creatinine-adjusted levels of ApoC3 were significantly higher in the $\mathrm{AD}+\mathrm{MCI}$ group relative to the control group $(p=$ $0.015)$. When MCI samples were excluded, the $p$ value became smaller $(p=0.011)$. Receiver operating characteristic analysis was performed for ApoC3 and the sensitivity, specificity, and area under the curve are shown in Figure 4. 
Fig. 4. Receiver operating characteristic curve of ApoC3 between the AD (without MCI) and control groups. AD, Alzheimer's disease; ApoC3, apolipoprotein C3; AUC, area under the curve; $\mathrm{CI}$, confidence interval; $\mathrm{MCI}$, mild cognitive impairment.

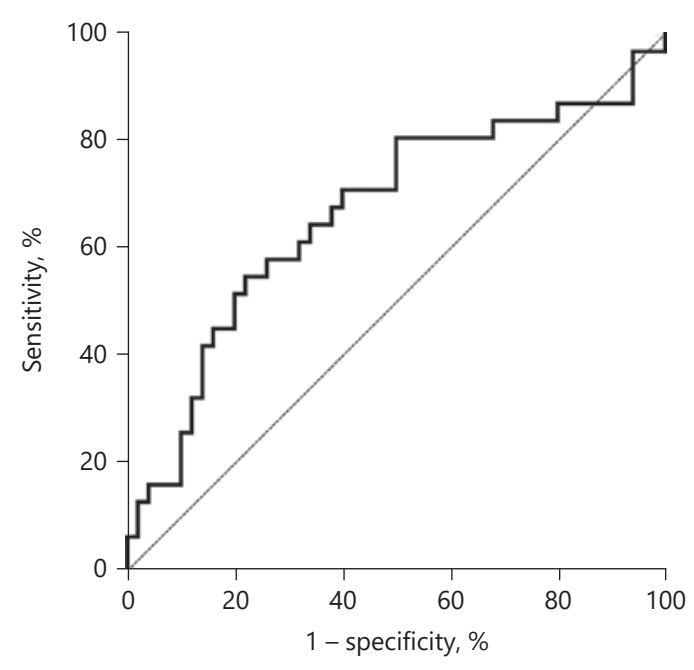

AUC (95\% Cl): 0.668 (0.540-0.795)

\section{Discussion}

We previously conducted a comparative study of urinary proteomes of AD patients and cognitively normal elderly people using label-free mass spectrometry [7]. In the present study, the results from the previous study were confirmed and validated in two independent sample sets. The candidate proteins examined were ApoC3, Igfbp3, and ApoD, which were most significantly increased or decreased in the label-free quantification.

Confirmation of the label-free quantification of mass spectrometry results was performed with the same sample set used in the earlier proteomics study (set 1). Candidate proteins for validation were selected based on their statistical significance and abundance in urine, given reports suggesting that proteins that are more abundant are generally easier to precisely quantify in label-free quantification, while proteins of low abundance show greater variation $[12,13]$. The results of the ELISA for ApoD, an abundant lipoprotein in urine [13], were similar to those in the earlier proteomics study. Moreover, the results of the ELISA for Igfbp3, which was the most significantly increased protein in the AD group in the proteomics study but was low in abundance in urine, were also consistent with those from label-free quantification. In contrast, the results of the ELISA for ApoC3, the most significantly decreased protein in the $\mathrm{AD}$ group in the proteomics study and among the lowest in abundance in urine, showed an opposite tendency relative to the results of label-free quantification. Higher sample numbers may be required to accurately detect changes in low-abundance proteins.

The results of set 1 were confirmed using an independent sample set (set 2). The ELISA results for Igfbp3 and ApoD in sets 1 and 2 were inconsistent. One possible reason for this may be the large interindividual variation in the composition of urine [12-17]. Another reason may be sampling bias. All urine samples of $\mathrm{AD}$ (and $\mathrm{MCI}$ ) patients were obtained from a hospital, while the control urine samples of sets 1 and 2 were obtained from communitydwelling elderly volunteers and a hospital, respectively. This may explain the high percentage of control participants with diabetes in set 2 (Table 1).

Interestingly, the ELISA results for ApoC3 were similar between sets 1 and 2. When combining the results of both, ApoC3 levels were significantly higher in the $\mathrm{AD}+\mathrm{MCI}$ group relative to the control group (Fig. 3a). The difference was even more significant when MCI samples were excluded from the analysis (Fig. 3d). 
ApoC3 is an abundant apolipoprotein mainly present in triglyceride-rich lipoproteins (chylomicrons and VLDL) and, to a lesser extent, in LDL and HDL particles [18]. ApoC3 is primarily synthesized in the liver [18], but is also present in the brain [19]. Epidemiological studies have found that ApoC3 is associated with a higher risk of cardiovascular disease and type 2 diabetes $[20,21]$. ApoC3 affects the development of cardiovascular disease by regulating triglyceride metabolism and through its direct atherogenic effects [22]. There is also evidence that ApoC3 exerts proinflammatory effects on both endothelial cells and monocytes [23-25].

Dysregulation of lipid homeostasis and inflammation underlie the development of AD pathology [26-28], and type 2 diabetes increases the risk of AD [29]. However, studies on the association between ApoC3 and risk of $\mathrm{AD}$ are limited. Two cross-sectional studies found lower levels of plasma ApoC3 in AD patients compared to control participants [30,31]. A case-cohort study with a mean follow-up period of 5.1 years associated higher levels of plasma ApoC3 with lower dementia risk, but not after adjusting for APOE $\varepsilon 4$ carrier status [32]. A cohort study with 2 years of follow-up concluded that plasma levels of ApoC3 did not predict future cognitive decline and also found lower plasma ApoC3 levels in APOE \&4 carriers; increased plasma ApoC3 levels were associated with a decrease in grey matter volume [33]. In another study, the prevalence of homozygosity for the $-641 \mathrm{C}$ allele in the APOC3 promoter (rs2542052) was significantly higher in centenarians than controls, and this genotype was associated with significantly lower serum levels of ApoC3 [34]. The APOE genotype of participants was not available in the current study, so similar analyses could not be performed.

Recently, Zewinger et al. [35] identified ApoC3 as an endogenous mediator that induces NOD-like receptor protein 3 (NLRP3) inflammasome-dependent sterile inflammation. Inflammasomes are multiprotein complexes that mediate proteolytic activation of IL-1 $\beta$ and IL-18 [36]. Zewinger et al. [35] treated human monocytes with HDL, LDL, and VLDL, but only VLDL stimulated the release of IL-1 $\beta$. Among the three most abundant proteins in VLDL particles, only ApoC3 induced IL-1 $\beta$ release [35]. In the brain, NLRP3 is predominantly located in microglia [37]. Amyloid beta accumulation in AD brains reportedly activates the microglial NLRP3 inflammasome. These studies suggest that ApoC3 should be further investigated as a potential urinary biomarker for AD.

In conclusion, we confirmed that ApoC3 may have potential as a urinary biomarker for AD. Further analysis with a larger sample size in a prospective setting is warranted to explore this possibility.

\section{Acknowledgement}

The authors thank all study participants and the following institutions for their contributions: Shibata Comprehensive Health Care Service Center and NCGG Biobank. They also thank Dr. B. Xu, Dr. S. Saito, and all members of the Biofluid Biomarker Center, Niigata University, for their invaluable help.

\section{Statement of Ethics}

This study was approved by the Human Research Ethics Committee of Niigata University (approval number: 1836, 2015-2081) and by the Committee of Ethics of Human Research of the NCGG (approval number: 2017-0357). All participants from Niigata University Hospital and the NCGG Biobank signed informed consent forms, and all participants of the Murakami cohort [10] were informed through a verbal consent process. 


\section{Conflict of Interest Statement}

The authors have no conflicts of interest to declare.

\section{Funding Sources}

Financial support was provided in part by JSPS KAKENHI (grants No. JP23249035, JP15H04782, and JP17K19799 to K. Nakamura and No. JP16K09051 to Y. Watanabe); a grant from SENSHIN Medical Research Foundation to Y. Watanabe; a grant from the Japan Agency for Medical Research and Development (grant No. 18dm0107143 to T. Ikeuchi); and a grant from the Center of Innovation Program from the Ministry of Education, Culture, Sports, Science, and Technology to T. Yamamoto.

\section{Author Contributions}

Y. Watanabe conducted the study, collected the data, preformed the statistical analysis, interpreted the data, and wrote the manuscript. Y. Hirao conducted the study, preformed the data analysis, and wrote and revised the manuscript. K. Kasuga, T. Tokutake, K. Kitamura, and S. Niida collected the data, interpreted the results, and revised the manuscript. T. Ikeuchi advised on the study design, supervised the data collection, and contributed to manuscript writing and revision. K. Nakamura conceived the study, supervised the data collection, advised on the statistical analysis, and critically revised the manuscript. T. Yamamoto conceived the study, supervised the data collection, advised on the data analysis, and critically revised the manuscript. All authors read and approved the final manuscript.

\section{References}

1 WHO. Dementia fact sheet. 2019. Available from: www.who.int/news-room/fact-sheets/detail/dementia [cited January 24, 2020].

2 Livingston G, Sommerlad A, Orgeta V, Costafreda SG, Huntley J, Ames D, et al. Dementia prevention, intervention, and care. Lancet. 2017 Dec;390(10113):2673-734.

3 Kivipelto M, Mangialasche F, Ngandu T. Lifestyle interventions to prevent cognitive impairment, dementia and Alzheimer disease. Nat Rev Neurol. 2018 Nov;14(11):653-66.

4 Khoury R, Ghossoub E. Diagnostic biomarkers of Alzheimer's disease: A state-of-the-art review. Biomark Neuropsychiatry. 2019 Dec;1:100005.

5 Thomas S, Hao L, Ricke WA, Li L. Biomarker discovery in mass spectrometry-based urinary proteomics. Proteomics Clin Appl. 2016 Apr;10(4):358-70.

6 Ruan Q, D'Onofrio G, Sancarlo D, Greco A, Yu Z. Potential fluid biomarkers for pathological brain changes in Alzheimer's disease: implication for the screening of cognitive frailty. Mol Med Rep. 2016 Oct;14(4):3184-98.

7 Watanabe Y, Hirao Y, Kasuga K, Tokutake T, Semizu Y, Kitamura K, et al. Molecular Network Analysis of the Urinary Proteome of Alzheimer's Disease Patients. Dement Geriatr Cogn Disord Extra. 2019 Feb;9(1):53-65.

8 Yao F, Hong X, Li S, Zhang Y, Zhao Q, Du W, et al. Urine-Based Biomarkers for Alzheimer's Disease Identified Through Coupling Computational and Experimental Methods. J Alzheimers Dis. 2018;65(2):421-31.

9 Nakamura K, Takachi R, Kitamura K, Saito T, Kobayashi R, Oshiki R, et al. The Murakami Cohort Study of vitamin $\mathrm{D}$ for the prevention of musculoskeletal and other age-related diseases: a study protocol. Environ Health Prev Med. 2018 Jun;23(1):28.

10 Sugishita M, Koshizuka Y, Sudou S, Sugishita K, Hemmi I, Karasawa H, et al. The validity and reliability of the Japanese version of the Mini-Mental State Examination (MMSE-J) with the original procedure of the Attention and Calculation Task (2001). Jpn J Cogn Neurosci. 2018;20(2):91-110.

11 Griffin NM, Yu J, Long F, Oh P, Shore S, Li Y, et al. Label-free, normalized quantification of complex mass spectrometry data for proteomic analysis. Nat Biotechnol. 2010 Jan;28(1):83-9.

12 Liu X, Shao C, Wei L, Duan J, Wu S, Li X, et al. An individual urinary proteome analysis in normal human beings to define the minimal sample number to represent the normal urinary proteome. Proteome Sci. 2012 Nov; 10(1):70. 
13 Nagaraj N, Mann M. Quantitative analysis of the intra- and inter-individual variability of the normal urinary proteome. J Proteome Res. 2011 Feb;10(2):637-45.

14 Sun W, Chen Y, Li F, Zhang L, Yang R, Zhang Z, et al. Dynamic urinary proteomic analysis reveals stable proteins to be potential biomarkers. Proteomics Clin Appl. 2009 Mar;3(3):370-82.

15 He W, Huang C, Luo G, Dal Prà I, Feng J, Chen W, et al. A stable panel comprising 18 urinary proteins in the human healthy population. Proteomics. 2012 Apr;12(7):1059-72.

16 Shao C, Wang Y, Gao Y. Applications of urinary proteomics in biomarker discovery. Sci China Life Sci. 2011 May; 54(5):409-17.

17 Shao C, Zhao M, Chen X, Sun H, Yang Y, Xiao X, et al. Comprehensive Analysis of Individual Variation in the Urinary Proteome Revealed Significant Gender Differences. Mol Cell Proteomics. 2019 Jun;18(6):1110-22.

18 Taskinen MR, Borén J. Why Is Apolipoprotein CIII Emerging as a Novel Therapeutic Target to Reduce the Burden of Cardiovascular Disease? Curr Atheroscler Rep. 2016 Oct;18(10):59.

19 Elliott DA, Weickert CS, Garner B. Apolipoproteins in the brain: implications for neurological and psychiatric disorders. Clin Lipidol. 2010 Aug;51(4):555-73.

20 Brahimaj A, Ligthart S, Ikram MA, Hofman A, Franco OH, Sijbrands EJ, et al. Serum Levels of Apolipoproteins and Incident Type 2 Diabetes: A Prospective Cohort Study. Diabetes Care. 2017 Mar;40(3):346-51.

21 Wyler von Ballmoos MC, Haring B, Sacks FM. The risk of cardiovascular events with increased apolipoprotein CIII: A systematic review and meta-analysis. J Clin Lipidol. 2015 Jul-Aug;9(4):498-510.

22 Luo M, Peng D. The emerging role of apolipoprotein C-III: beyond effects on triglyceride metabolism. Lipids Health Dis. 2016 Oct;15(1):184.

23 Kawakami A, Aikawa M, Libby P, Alcaide P, Luscinskas FW, Sacks FM. Apolipoprotein CIII in apolipoprotein B lipoproteins enhances the adhesion of human monocytic cells to endothelial cells. Circulation. 2006 Feb; 113(5):691-700

24 Kawakami A, Aikawa M, Alcaide P, Luscinskas FW, Libby P, Sacks FM. Apolipoprotein CIII induces expression of vascular cell adhesion molecule- 1 in vascular endothelial cells and increases adhesion of monocytic cells. Circulation. 2006 Aug;114(7):681-7.

25 Kawakami A, Aikawa M, Nitta N, Yoshida M, Libby P, Sacks FM. Apolipoprotein CIII-induced THP-1 cell adhesion to endothelial cells involves pertussis toxin-sensitive $\mathrm{G}$ protein- and protein kinase $\mathrm{C} \alpha$-mediated nuclear factor-kappaB activation. Arterioscler Thromb Vasc Biol. 2007 Jan;27(1):219-25.

26 Zhang ZG, Li Y, Ng CT, Song YQ. Inflammation in Alzheimer's Disease and Molecular Genetics: recent Update. Arch Immunol Ther Exp (Warsz). 2015 Oct;63(5):333-44.

27 Kao YC, Ho PC, Tu YK, Jou IM, Tsai KJ. Lipids and Alzheimer’s Disease. Int J Mol Sci. 2020 Feb;21(4):1505.

28 Jung YJ, Kim YH, Bhalla M, Lee SB, Seo J. Genomics: New Light on Alzheimer's Disease Research. Int J Mol Sci. 2018 Nov; 19(12):3771.

29 Paul KC, Jerrett M, Ritz B. Type 2 Diabetes Mellitus and Alzheimer's Disease: Overlapping Biologic Mechanisms and Environmental Risk Factors. Curr Environ Health Rep. 2018 Mar;5(1):44-58.

30 Shih YH, Tsai KJ, Lee CW, Shiesh SC, Chen WT, Pai MC, et al. Apolipoprotein C-III is an amyloid- $\beta$-binding protein and an early marker for Alzheimer's disease. J Alzheimers Dis. 2014;41(3):855-65.

31 Lin Q, Cao Y, Gao J. Decreased expression of the APOA1-APOC3-APOA4 gene cluster is associated with risk of Alzheimer's disease. Drug Des Devel Ther. 2015 Sep; 9:5421-31.

32 Koch M, DeKosky ST, Goodman M, Sun J, Furtado JD, Fitzpatrick AL, et al. High density lipoprotein and its apolipoprotein-defined subspecies and risk of dementia. J Lipid Res. 2020 Mar;61(3):445-54.

33 Song F, Poljak A, Crawford J, Kochan NA, Wen W, Cameron B, et al. Plasma Apolipoprotein Levels Are Associated with Cognitive Status and Decline in a Community Cohort of Older Individuals. PLoS One. 2012; $7(6): e 34078$.

34 Atzmon G, Rincon M, Schechter CB, Shuldiner AR, Lipton RB, Bergman A, et al. Lipoprotein genotype and conserved pathway for exceptional longevity in humans. PLoS Biol. 2006 Apr;4(4):e113.

35 Zewinger S, Reiser J, Jankowski V, Alansary D, Hahm E, Triem S, et al. Apolipoprotein C3 induces inflammation and organ damage by alternative inflammasome activation. Nat Immunol. 2020 Jan;21(1):30-41.

36 Dionisio-Santos DA, Olschowka JA, O'Banion MK. Exploiting microglial and peripheral immune cell crosstalk to treat Alzheimer's disease. J Neuroinflammation. 2019 Apr;16(1):74.

37 Heneka MT, McManus RM, Latz E. Inflammasome signalling in brain function and neurodegenerative disease. Nat Rev Neurosci. 2018 Oct;19(10):610-21. 